\title{
The Role of Context for Recommendations in Digital Libraries
}

\author{
Zohreh Dehghani Champiri, Siti Salwah Binti Salim, and Seyed Reza Shahamiri
}

\begin{abstract}
This paper discusses the role of context in making recommender systems for digital libraries. It first introduces different types of recommender systems then discusses the role of context in making recommendations for users searching in digital libraries. This study highlights the important role of context and the need for more investigation on context-aware recommender system since exploiting contextual information in recommender systems is an effective approach to create more accurate and relevant recommendations. The article stresses the need for qualitative research on the subject in order to find out the elements that constitute the context from the users' viewpoint and the way a context-aware recommender system could be effectively designed. Past studies seem to have failed to survey on enough attention to the role of context in designing recommender systems for digital libraries.
\end{abstract}

Index Terms-Recommender system, digital library, context, information seeking in context.

\section{INTRODUCTION}

Today, access to useful and relevant information is a prevalent problem due to information overload [1]. Recommender Systems (RSs) are a special class of personalized systems that aim at predicting a user's interest in available products and services by relying on information seeking behavior and previously rated items or item features [1] . On the whole, they are mainly intelligent applications, created to support users by personalized recommendations in search process and their decision-making while interacting with large information spaces.

RSs are used in digital libraries, electronic stores, travel tours, restaurants, hospitals and in general can be useful in any decision-making process to provide predictions of appropriate items to specific users [2]. Moreover, dramatic increase in unorganized information on the web has made such systems a prerequisite for the information seeking on the

World Wide Web. A study of 96 RSs by Rao and Talwa [3] showed that RSs have been developed in various domains including web recommendation application, movie/TV, information/document recommendation, Usenet news recommendation, information filtering and sharing, music recommendation, restaurant recommendation application,

Manuscript received June 10, 2014; revised August 12, 2014.

Zohreh Dehghani Champiri and Siti Salwah Binti Salim are with the Department of Software Engineering, Faculty of Computer Science and Information Technology, University of Malaya, Iran (e-mail: z.dehghani@yahoo.com, salwa@um.edu.my).

Seyed Reza Shahamiri with Faculty of Business and Information Technology, Manukau Institute of Technology, Auckland, New Zealand (e-mail: rshahamiri@gmail.com). organizational expertise recommendation, personalized newspaper domain, e-commerce application, travel recommendation, electronic catalogue item recommendation, learning resources recommendation, Web search and filtering, virtual fair recommendation, jokes recommendation, and image RSs. Also a taxonomy and comprehensive explanation of elements in recommender agents on the Internet has been provided by Montaner, Lopez and De la Rosa [4].

During a commercial interaction, RSs have advantages for both customers and merchants. For example, in a business interaction through the online shopping, RSs recommend customers items so help customers to find their favorite items among an overwhelming number of items in an electronic department store; therefore RSs can facilitate and accelerate shopping for users. Also, merchants proffer their products and hereby they can increase their sales and customers satisfaction by offering the new and preferable items.

Similarly, in a digital library, RSs can manage information overload by helping users to choose appropriate information items from a large set of alternatives [5]. Information users normally favor recommendations. Traditional library users tend to check the returned books basket and choose from the returned books assuming that those books that had been read by others are more likely to be good. This could be considered some sort of recommendation. Past research has shown that scientists also find a considerable number of papers they read through recommendations made by their colleagues.

RSs can help overcome information overload problem by exposing users to interesting, novel, surprising and relevant items [7], [8] based on preferences users have expressed either explicitly or implicitly [9]. They can introduce users to new items that have not been known or have not been retrieved. Hence recommendations can assist users in meeting their information needs [10]. From the appearance of RSs as an independent research area in the mid- 1990s [11], [12], many studies have been done on RSs in different domains [13]. Several studies have been done on them due to their effectiveness and performance in difference applications [14] however the issue of creating more accurate and relevant recommendations for users is still open and significant [15], [16]. In recent years, studies on RSs have been actuated to use contextual information such as location, time, and task in order to make more relevant and personalised recommendations [11], [12]. In this article, we first introduce different types of RSs and their weaknesses and strengths. Then we discuss their applications in digital libraries with regard to some of the definitions of digital libraries. Finally, we shall discuss the role that context can play in designing RSs for digital libraries. 


\section{RECOMMENDATION APPROACHES}

Making suggestions is a common process in everyday life. Suggestions and recommendations for reading a paper, selecting a special restaurant, buying some books or goods and seeing a doctor are just a few examples to mention. The question which may come to mind is what factors do people consider when they recommend something to each other?

Approaches which are used for making recommendations by RSs are mainly based on people's real behaviors in daily life.

RSs provide recommendation via different approaches, but the most common systems fall into three main classes: Collaborative Filtering, Content- Based, and Knowledge -Based. There is another class that is a combination of two or all of these three approaches and is called Hybrid. A classification of RSs research based on their approaches was provided by Adomavicius and Tuzhilin [17]. In the following we briefly describe recommending approaches.

Collaborative Filtering(CF): $\mathrm{CF}$ approach is the most common approach for creating recommendations [14]. CF is based on the premise that users looking for information should be able to make use of what others have already found and evaluated. So collaborative filters support users to choose based on the opinions and preferences of other users, it can be used to facilitate links between users with similar interests [18].

CF approaches employ users' behavior similarities and users' functional patterns to create recommendations [19], [20]. In these approaches, statistical analysis and modeling are made by data mining in databases, browsing and controlling of users' behavior, items ratings by various users, survey of users history and interest profiles. The ratings which are given to items have obtained either explicitly from users or implicitly from observations of users' behavior [18], [21]. As mentioned, in CF approach users behaviors and history of their preferences are monitored and recommendations are offered to new users by finding the nearest neighbors [20], [21]. The nearest neighbors are more similar to new user. New user who receives recommendations is called "target user". We explain it by a simple example; as shown in Table I, both User 2 and User 3 have bought (or have read) Book 2 and Book 3. So they are similar users or so-called "User 3 is in the neighborhood of User 2". Therefore book 5, which had been selected by User 2 , may be a good recommendation for the User 3 with the probability that Book 5 is User 3's preferences or interests.

TABLE I: COLLABORATIVE FILTERING APPROACH

\begin{tabular}{|l|l|l|l|l|l|l|}
\hline & Book 1 & Book 2 & Book 3 & Book 4 & Book 5 & Book 6 \\
\hline User 1 & $\checkmark$ & & & $\checkmark$ & & $\checkmark$ \\
\hline User 2 & & $\checkmark$ & $\checkmark$ & & $\checkmark$ & \\
\hline User 3 & & $\checkmark$ & $\checkmark$ & & & \\
\hline User 4 & & $\checkmark$ & & & & $\checkmark$ \\
\hline
\end{tabular}

Studies showed that $\mathrm{CF}$ approach is a critical approach in many RSs inasmuch as they lead to increment of quality of recommendations. In $\mathrm{CF}$, preferences of identified users are leveraged to similar users [22]. CF approaches are often classified into two categories as Memory-based and
Model-based. In the Memory-based CF approach, RSs make recommendations using all rating data retained in the memory [18], [23]. So the similarities between users are identified by memorized rating and the required predictions are computed on demand. In contrast to the Memory-based approach, in the Model-based approach, RSs use historical data to make models capable of predicting the ratings for items that a new user has not rated before. So, similarities between users are identified by designed models such as clustering models and statistical models.

Content-Based $(C B)$ : The root of $\mathrm{CB}$ approach can be stemmed back to Information Retrieval (IR) [20]. In the CB approaches, similarities and relations between items in a database are identified by cataloging, content analysis, keyword identification and tagging and then recommendations are made based on semantic relations between the content of the items and the preferences that a user has expressed in the past [24].

$\mathrm{CB}$ approaches focus on finding correlations between content of items as opposed to correlation between users as is the case in CF approaches. CB approach is employed where the items can be evaluated (or rated) by keywords such as textual documents and web pages (HTML) [18], [20]. Recommendations are often made by comparing users' profiles and their preferences with keywords generated through content analysis of documents. User's preferences can be identified implicitly or explicitly. In the explicit method it is necessary to evaluate documents with a specific criterion. In the implicit method user's preferences are obtained by observing user behavior [20]. While the latter is more favored by users, its implementation is more difficult. Porter-stemmed Term Frequency/Inverse Document Frequency (TF/IDF) is one the most common and well-known CB filtering methods. In this method the full-text of a document is used in order to generate recommendations. In TF/IDF the frequency of stemmed words is counted and compared to the entire corpus then recommendations are created by matching the items with the important keywords. As mentioned before, information retrieval fundamentals are core of the CB approaches such as TF/IDF [18].

Knowledge-Based $(K B)$ : The third approach is $\mathrm{KB}$ approach that employs knowledge about users and products (items) to generate recommendations. So RSs offer recommendations based on the knowledge of user's needs and items[25]. KB systems use knowledge analyzing methods such as Neurotic networks, fuzzy- genetic algorithms, decision trees, and case base reasoning [26]. The case base reasoning method is the most prevalent method for knowledge analyzing [27].

Hybrid: According to studies, each of the above mentioned approaches has its strengths and weaknesses [27]-[30]. A CF system collects more ratings from more users; this increases the probability of someone in the system being a good match for any given new user [30]. However, a CF system must be initialized with a large amount of data, because a system with a small base of ratings is unlikely to be very helpful. Further, the accuracy of the system is very sensitive to the number of rated items that can be associated with a given user [28]. Therefore, CF approaches can be useful provided a superabundant number of users' behaviors has been 
identified as well as an adequate number of rated items has been accounted [30].

In CB approaches some problems may occur. Typically, until user has not rated many items, useful clustering cannot be generated for recommendations. In this situation, some systems employ the nearest-neighbor's ratings for the new user to overcome this problem although the system have not offered any recommendations because the recommendations have been based on ratings it has not a particular user ratings [24], [28]. Besides, CB approaches are extremely dependent on the content and if the content does not contain adequate information to distinguish items the user prefer from items the user does not prefer, no helpful recommendation can be made [31].

In KB RSs, the above problems do not exist as the generation of recommendations does not rely on user ratings and also information gathering about a particular user is not necessary [27], [32].The KB approaches rely on knowledge obtained about items and users [30]. As it has been noted, in this approach knowledge is acquired using some analytical methods such as decision trees and fuzzy- genetic algorithms. Due to these distinguished characteristics, KB RSs are valuable and supplementary to other types of RSs [27].

Since each approach has some shortcomings, some RSs' designers employ a Hybrid approach which is the combination of two or more approaches mentioned above. The Hybrid approach is utilized to decrease the limitations and increase the system efficiency. It is necessary to mention, $\mathrm{CF}$ is the main method which is combined by other approaches in the Hybrid approach [28].

\section{RSS IN DIGITAL LIBRARIES}

Today, people have most of their needed information at their fingertips. Users usually input keywords to retrieve desired information but studies have showed that the retrieved results are not always what they expect [33]. Therefore, some tools like RSs have been developed to support users in information seeking and decision making [34]. They recommend appropriate resources or suitable services to users based on their interests and preferences [35].

RSs in digital library can assist members who are not familiar with search tools, strategies or are unable for any reason to retrieve satisfactory results, hence RSs can be an assistant or adviser for users in digital library as a librarian and hereby they can facilitate and accelerate the search process. Besides, RSs can be useful in other parts of library services, such as collection acquisition and library materials selection. For instance, director of library can provide materials for users based on the recommendations created by RSs because recommendations represent the users' preferences and interests.

The way we define a digital library has a fundamental impact on design of RSs since the services, goals and users' information seeking vary in different library contexts.

Since 1990s, the term of library has been accompanied by various terms such as digital, electronic, virtual, online, cyber accordingly various definitions have been emerged while it seems that these terms have been used synonymously and there is no significant difference between them [36]. Between 1972 and 1995, Howard D. White and Kate McCain's [37] reviewed and analyzed the structure of the information science discipline. The results showed that many new areas such as information retrieval and user-system relationships; bibliometrics; automated library systems and online catalogs; science communication; and user theory have been appeared in information science discipline which the new researches on digital library have originated from here [38].

Several definitions have been posed by authors such as Arms; Fox; Lesk; Tedd and Large [39]. The definitions have been derived from different perspectives such as; Computer Science, Library and Information Science, Commercial Enterprises, Politicians and Governments perspectives. We indicate three definitions of digital libraries in following. We discuss three definitions here and give the features of digital library in order to design an applicable RS for users.

The first definition is one of the most cited definitions which stated by "Digital Library Federation (DLF)" and is avilable at the DLF site (1998). In the this definition, digital library has been considered as an organization which promises to prepare various services for users regardless of users' differentiation while indicates that "Digital libraries are organizations that provide the resources, including the specialized staff, to select, structure, offer intellectual access to, interpret, distribute, preserve the integrity of, and ensure the persistence over time of collections of digital works so that they are readily and economically available for use by a defined community or set of communities".

From a Library and Information Science perspective, Christine L. Borgman [40] points out: "Digital libraries are a set of electronic resources and associated technical capabilities for creating, searching, and using information. In this sense they are an extension of information storage and retrieval systems that manipulate digital data in any medium (text, images, sounds; static or dynamic images) and exist in distributed networks". She mentioned that "data that describe various aspects of the data and metadata that consist of links or relationships to other data and metadata are considered the content of digital libraries"

And finally the third definition has been indicated from the Computer Science point of view by William Y. Arms where he illustrates "In computing terminology, a distributed system is a group of computers that work as a team to provide services to users. Digital libraries are some of the most complex and ambitious distributed systems ever built. The term "digital libraries" covers the creation and distribution of all types of information over networks, ranging from converted historical materials to kinds of information that have no analogues in the physical world" [36]

Indeed digital libraries are complex information systems, under evolution and development, and their acceptance by users is of critical importance to ensure their correct evolution. The success of digital libraries depends increasingly on their ability to provide differentiating features and value-added services for their users [35]. Hence, for making useful recommendations, we need first know that what users really want and expect a digital libray and its services. For instance, Ackerman \& Mainwaring stated that because of users' privacy issues, users and are not eager to 
use RSs in digital libraries [41]. So for desinging RSs, we require to explor the contexts that users are seeking infromation and clarify which context is useful for making recommendation, when and how it can be applied.

\section{The Role OF CONTEXT FOR RECOMMENDATIONS}

Many disciplines are involved in creating recommendation systems including Computer Science, Social Science, Cognitive Science, Business Science, Linguistic Science, Information Science and other related fields which make such systems an interdisciplinary research area [16]. At present, contextual information has been employed as a main source for the accuracy of predictions in RSs [42]. Several studies have shown that using contextual information in making recommendation can increase the performance of RSs [11], [ 17], [43].

Some studies emphasize on contextual information for creation of recommendation versus classical approaches or non- contextual approaches such as CF, CB and KB [44]. Recently, contextual information modelling has been applied to improve classical recommendations in order to determine the status of status of people, places, objects and devices in the environment and improve the accuracy of recommendations. However, identifying and applying valid contextual information for different domains and users are challenges that should be addressed [45] these challenges have become a paradigm shift in recommending approaches in the recent years [16].

What is contextual information?

In everyday life, when people communicate with each other, they can comprehend a lot of emblems of their situations implicitly or explicitly. While people perceive emblems through an interaction, they analyze and interpret them, compare and interlace to their suppositions, pre-knowledge and pre-imageries then conclude and react verbally or nonverbally whereby they can bandy their ideas. These emblems, so-called "context" and application that utilizes context to provide relevant information to users, called Context-Aware Application. The term "context" emerged in computer science in the late 1980s [46], and the idea of context awareness in computing was introduced by Schilit in 1994 [47]. When people interact with computers, they expect to encounter proper reactions from computers, unfortunately computers are not able to retort as human. Therefore, it is required to simulate human-computer interaction like human interaction as far as possible. From Dey's view, computer's access to context is led to increase in the richness of communication subsequently it provides more useful computational services [48]. Similar to digital library, many definitions have been proposed for the context from many viewpoints and there is not yet any consensus on what exactly context is [11]. For example, Bazire and Brézillon [49] tried to identify the main components of the context on the basis of the analysis of a corpus of 150 definitions coming mainly from the web in different domains of cognitive sciences and close disciplines. They analyzed the definitions and concluded that all definitions can be presented by parameters such as constraint, influence, behavior, nature, structure and system. They mentioned that it is not easy to present an unanimous definition of context since context is very ill-defined concept however they try to propose a model of context presenting the components of a situation (where the context is considering) and the relations and links between the components (see Fig. 1).

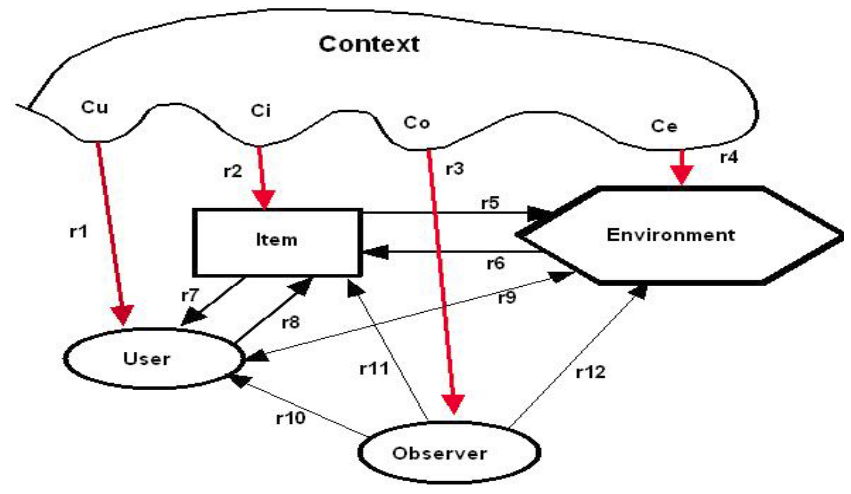

Fig. 1. Context model presented by bazire and brézillon [49].

Here, we mention a few of the definitions that could be helpful for our discussion on context- aware RSs.

Schilit \& Theimer [50] define the context as location, identity, nearby people and objects. In a similar definition by Brown, context constitutes of location, identity, nearby people and objects and Season. In another definition, Pascoe [51] indicates that context respond to the following questions: Where are you? Who you are with? What resources are nearby?

One of the most cited definitions from a computer science viewpoint was offered by Dey and Abowd [52]. They express that the context is "any information that can be used to characterize the situation of an entity and they categorized the context into four dimensions: location, identity, time and activity". In Dey and Abowd's definition there are two context levels: primary contexts which are four mentioned dimensions and secondary contexts gained from primary contexts. Many pieces of related information such as phone numbers, addresses, email addresses, birthrate, etc. can be acquired from an entity's location. This information that is acquired from primary contexts is numerated as secondary contexts [52].

What is contextual information in digital libraries?

To clarify the above question, imagine the following scenario;

Two users refer to librarian and ask resources about "Urban Traffic". One of them is undergraduate student and needs information for his assignment and the other one is graduate student and needs resources for writing a research project. The first user seems nervous and worried and the second user who has stood on the corner of library works at a Construction Company. Besides, the library is very crowded and librarian is too busy. She has to answer other users by telephone simultaneously. Considering the mentioned scenario:

1) What should the librarian do in this situation?

2) What resources does the librarian recommend to these users?

3) How does the librarian find the most appropriate 
resources for each of them?

In traditional libraries, librarians mostly try to match resources to users' queries or needs based on their knowledge and familiarity with a broad range of library resources. Sometimes, they have to obtain more information about users to find the exact users' information need such as their activity, task, background knowledge, experiences, topic, and purpose as well as conditions like time, location, situation, identity, and accessibility since some people cannot express their exact needs or do not ask for what they really want.

Obviously, the sources which are proposed by librarian to the above mentioned users are not necessarily similar because of their goals and other factors such as their background knowledge. Moreover, physical conditions and the environment where the interaction occurs play a substantial role in a successful interaction. A friendly, attractive environment will attract users and more questions with enthusiasm on the contrary a dark, crowded or unfriendly environment may repulse users.

People can perceive such factors directly or indirectly from surrounding or the situations they are but in digital environments, the users' situations cannot be understood easily because there is no face to face interaction. Moreover, computers are not able comprehend users' situations as similar as that occurs in real interaction and accordingly, these factors can be ignored while they have a considerable influence on a successful interaction between user is seeking information and system.

In interaction between users with digital library, RS can play the role of librarian in order to recommend right resources to right users therefore they not only need to be aware of users' contexts but also they should be aware of the problems which user is seeking information to solve them.

\section{CONCLUSION}

It seems that trying to reach a consensual definition for context which is applicable for all domains is an ineffectual effort because context is a concept from the real world and it is impossible to delimit its territories by giving a certain definition [16] .Moreover, people comprehend phenomena by an interpretive understanding which is dependent on the contexts they are. Hermeneutic theory widely discusses that contexts change the epistemological criteria because context evokes different impressions in each reader therefore some researches have mentioned that context is a dynamic theorem [53]. Hereby, two questions spring to mind:

1) How should we design and develop context-aware applications in different environments in order to ensure their applicability and usefulness for users?

2) What contexts should we consider in designing and making RSs in digital libraries?

Because of differences in the nature of interactions in different environments (e.g. academic digital libraries, e-shopping etc.), the question of context in RSs or other applications should be addressed in their own environments where they are used by their own users, also they need to be aware from users and their needs.

Several studies have used contextual information for enhancing recommendations in digital libraries [54]; however, not much progress has been made in the design and implementation of context-aware RSs for digital libraries and this area requires more deep investigations and studies in order to develop a real and tangible view to digital library services and their users [55], [56]. It seems that the lack of considerable progress of context-awareness in library services stems from two main issues:

First, the concept of context varies in different domains and requests separate studies on exploring the context in different systems such as digital libraries, E-learning, tourism. In most context- aware studies, context has been regarded as a general view and this indicates that studies have been done on context- aware RSs without a deep understanding of context. Besides, users have different seeking behaviors. Therefore, the concept of context in RSs should be demarcated in their own domains where they are used by the end users [57]. McNee [30] argued that users estimate information needs and formulate judgment criteria in their minds. Hence, in order to build relevant, useful, and effective recommender systems, the validity of contextual information through the eyes of users ought to be evaluated. Moreover, deep exploring of context in various applications may lead to a unified model as will be customizable for all applications regardless of their functionalities in the future. They all cause enhancing interactions between users and digital libraries.

The second issue is related to the research approaches which somehow addresses the above-mentioned issue. Researchers can obtain an in-depth understanding of user needs and their contexts by qualitative methods such as Ethnography, Grounded Theory, Discourse Analysis, Sense Making and other qualitative research methods. Qualitative research methods help researchers to understand people and the social and cultural contexts within which they live [58]. For enhancing the context-aware services, researchers now need to address questions such as: What does the user want? What is the user's problem? How has this problem formed? How does the user overcome the obstacles? What image does the user have of her or his situation? Finally, what type of assistance can the application offer to users in this situation? Qualitative research methods can answer such question as they emphasize on exploring a problem from viewpoint of its particular users in its particular context.

Generally, in development process, interdisciplinary concepts such as context are interpreted, analyzed and developed as well as evaluated by a technical insight or system oriented perspective which delimits human concepts to the technical knowledge capacities hence the users and their needs are formed or restructured base on the technical point of view. Ultimately, when users interact with context-aware applications, they do not feel comfortable give up using just because this is not what they want.

Finally, in our daily lives, we mostly accept and apply recommendations from people who are expert or at least we know them. Users interacting with RSs cannot easily accept the presented recommendations. They expect to receive useful recommendations from wise recommender so that they assume human personalities for RSs [30]. RSs can embed different personality characters in recommending such as considerate, risky, sensitive, temperamental, reasonable and 
stupid [56]. For example, if the system recommends romantic books to users while they are not interested or cannot understand any reason behind the recommendations, they may explains RS as a "helpless lover" [30] or if they constantly receive irrelevant recommendations, they assume that the recommender is stupid. The important issue is that they cannot trust RS as we cannot trust someone who gives us poor recommendations.

\section{REFRENCES}

[1] G. Lekakos and G. M. Giaglis, "Improving the prediction accuracy of recommendation algorithms," Approaches Anchored on Human Factors, Interacting with Computers, vol. 18, no. 3, pp. 410-431, 2006.

[2] K. Verbert, S. N. Lindstaedt, and D. Gillet, "Context-aware Recommender Systems," Journal of Universal Computer Science, vol. 16, no. 16, pp. 2175-2178, 2010.

[3] K. N. Rao, "Application domain and functional classification of recommender systems-a survey," Journal of Library and Information Technology, vol. 28, no. 3, pp. 17-35, 2010.

[4] M. Montaner, B. López, and J. L. D. L. Rosa, "A taxonomy of recommender agents on the internet," Artificial Intelligence Review, vol. 19 , no. 4, pp. 285-330, 2003.

[5] S. Gottwald and T. Koch, "Recommender systems for libraries" ACM Recommender Systems, Chicaog, IL, USA - nicht angenommen, 2011.

[6] H. R. Jamali and D. Nicholas, "Information-seeking behaviour of physicists and astronomers," Aslib Proceedings, Emerald Group Publishing Limited, 2008.

[7] G. Adomavicius et al., "Incorporating contextual information in recommender systems using a multidimensional approach," $A C M$ Transactions on Information Systems (TOIS), vol. 23, no. 1, pp. 103-145, 2005.

[8] A. Pommeranz et al., "Designing interfaces for explicit preference elicitation: a user-centered investigation of preference representation and elicitation process," User Modeling and User-Adapted Interaction, vol. 22, no. 4-5, pp. 357-397, 2012.

[9] L. Baltrunas et al., "Context relevance assessment and exploitation in mobile recommender systems," Personal and Ubiquitous Computing, vol. 16 , no. 5 , pp. 507-526, 2012.

[10] R. Sikka, A. Dhankhar, and C. Rana, "A Survey Paper on E-Learning Recommender System," International Journal of Computer Applications, vol. 47, no. 9, pp. 27-30, 2012.

[11] G. Adomavicius and A. Tuzhilin, Context-Aware Recommender Systems, in Recommender Systems Handbook, Springer, pp. 217-253, 2011.

[12] N. Y. Asabere, "Towards a viewpoint of context-aware recommender systems (CARS) and services," International Journal of Computer Science and Telecommunications, vol. 4, no. 1, pp.10-29, 2013.

[13] D. H. Park et al., "A literature review and classification of recommender systems research," Expert Systems with Applications, vol. 39, no. 11, pp. 10059-10072, 2012.

[14] B. Mehta and T. Hofmann, "A survey of attack-resistant collaborative filtering algorithms," IEEE Data Eng, Bull., vol. 31, no. 2, pp. 14-22, 2008.

[15] L. Liu, "The implication of context and criteria information in recommender systems as applied to the service domain," University of Manchester, 2013.

[16] G. Adomavicius and D. Jannach, "Preface to the special issue on context-aware recommender systems," User Modeling and User-Adapted Interaction, pp. 1-5, 2013

[17] G. Adomavicius and A. Tuzhilin, "Toward the next generation of recommender systems: A survey of the state-of-the-art and possible extensions," IEEE Transactions on Knowledge and Data Engineering, vol. 17, no. 6, pp. 734-749, 2005.

[18] P. Resnick et al., "GroupLens: an open architecture for collaborative filtering of netnews," in Proc. the 1994 ACM Conference on Computer Supported Cooperative Work, ACM, 1994.

[19] J. Herlocker, S. Jung, and J. G. Webster. (2012). Collaborative filtering for digital libraries. Technical Report. Oregon State University. [Online]. Available: http://hdl.handle.net/1957/28103

[20] M. Balabanović and Y. Shoham, "Fab: Content-based, collaborative recommendation," Communications of the ACM, vol. 40, no. 3, pp. 66-72, 1997.

[21] Z. Yuan, T. Yu, and J. Zhang, "A social tagging based collaborative filtering recommendation algorithm for digital library," in Proc. the 13th International Conference on Asia-Pacific Digital Libraries, pp. 192-201. 2011.

[22] P. Resnick and H. R. Varian, "Recommender systems," Communications of the ACM, vol. 40, no. 3, pp. 56-58, 1997.

[23] D. M. Pennock et al., "Collaborative filtering by personality diagnosis: A hybrid memory-and model-based approach," in Proc. the Sixteenth Conference on Uncertainty in Artificial Intelligence, Morgan Kaufmann Publishers Inc, 2000.

[24] M. J. Pazzani and D. Billsus, "Content-based recommendation systems," The Adaptive Web, Springer, pp. 325-341, 2007.

[25] B., Amini, R. Ibrahim, and M. S. Othman, "Discovering the impact of knowledge in recommender systems: a comparative study," International Journal of Computer Science and Engineering Survey, (IJCSES), vol. 2, no. 3, August 2011.

[26] A. Nakagawa and T. Ito, "An implementation of a knowledge recommendation system based on similarity among users' profiles," in Proc. the 41st SICE Annual Conference, IEEE, 2002.

[27] R. Burke, "Knowledge-based recommender systems," Encyclopedia of Library and Information Systems, vol. 69, no. 32, pp. 175-186, 2000.

[28] R. Burke, "Hybrid recommender systems: survey and experiments," User Modeling and User-Adapted Interaction, vol. 12, no. 4, pp. 331-370, 2002.

[29] A. S. Vivacqua, J. Oliveira, and J. M. de Souza, "I-ProSE: inferring user profiles in a scientific context," The Computer Journal, vol. 52, no. 7, pp. 789-798, 2009.

[30] S. M. McNee, "Meeting user information needs in recommender systems," Ph.D. Thesis, University of Minnesota, Minneapolis, MN, 2006.

[31] P. Lops, M. D. Gemmis, and G. Semeraro, "Content-based recommender systems: State of the art and trends," Recommender Systems Handbook, Springer, pp. 73-105, 2011.

[32] M. D. Gemmis et al., "Integrating tags in a semantic content-based recommender," in Proc. the 2008 ACM Conference on Recommender Systems, ACM, pp. 163-170, 2008.

[33] E. K. Zabihi, G. Ghinea, and S. Y. Chen, "Digital libraries: what do users want?" Online Information Review, vol. 30, no. 4, pp. 395-412, 2006.

[34] D. B. Krafft, A. Birkland, and E. J. Cramer, "Ncore: architecture and implementation of a flexible, collaborative digital library" in Proc the 8th ACM/IEEE-CS joint conference on Digital libraries, pp. 313-322, ACM, 2008

[35] A. Vellino and D. Zeber, "A hybrid, multi-dimensional recommender for journal articles in a scientific digital library," in Proc. the 2007 IEEE/WIC/ACM International Conference on Web Intelligence and International Conference on Intelligent Agent Technology, 2007.

[36] W.Y. Arms, "Digital libraries," MIT Press, Cambridge, 2001.

[37] H. D. White and K. W. McCain, "Visualizing a discipline: an author co-citation analysis of information science," Journal of the American Society for Information Science, vol. 49, no. 4, pp. 327-355, 1972-1995.

[38] W. Y. Arms, "The 1990s: the formative years of digital libraries," Library Hi Tech, vol. 30, no. 4, pp. 579-591, 2012.

[39] M. W. Harris. (2013). What's a digital library. [Online]. Available: http://next-nexus.info/writing/infostudies/digital_libraries.php

[40] C. L. Borgman, What are digital libraries? Competing Visions, Inf. Process. Manage, vol. 35, no. 3, pp. 227-243, 1999.

[41] M. Ackerman and S. Mainwaring, "Privacy issues and human-computer interaction," in Security and Usability: Designing Secure Systems that People Can Use, Sebastopol, CA, USA: O'Reilly, pp. 381-400, 2005.

[42] L. Baltrunas, "Exploiting contextual information in recommender systems" in Proc. the 2008 ACM Conference on Recommender Systems, pp 295-298, 2008.

[43] U. Panniello et al., "Experimental comparison of pre-vs. post-filtering approaches in context-aware recommender systems," in Proc. the third ACM Conference on Recommender Systems, ACM, 2009.

[44] L. Baltrunas and F. Ricci, "Context-based splitting of item ratings in collaborative filtering," in Proc. the third ACM Conference on Recommender Systems, pp. 245-248, New York, ACM, 2009.

[45] Z. Yujie and W. Licai, "Some challenges for context-aware recommender systems," in Proc. the 5th International Conference on Computer Science and Education (ICCSE), IEEE, pp. 362-365, 2010.

[46] J.-Y. Hong, E.-H. Suh, and S.-J. Kim, "Context-aware systems: a literature review and classification," Expert Systems with Applications, vol. 36, no. 4, pp. 8509-8522, 2009.

[47] P. J. Brown, J. D. Bovey, and X. Chen, "Context-aware applications: from the laboratory to the marketplace," IEEE Personal Communications, vol. 4, no. 5, pp. 58-64, 1997. 
[48] A. K. Dey, "Understanding and using context. Personal and ubiquitous computing," Personal and Ubiquitous Computing, Special issue on Situated Interaction and Ubiquitous Com- puting, vol. 5, no. 1, 2001.

[49] M. Bazire and P. Brézillon, "Understanding context before using it, in Modeling and using context," in Proc. the 5th in-ternational and Interdisciplinary Conference, Context, pp. 29-40, 2005.

[50] N. Schilit and M. Theimer, "Disseminating active map information to mobile hosts," Network, IEEE, vol. 8, no. 5, pp. 22-32, 1994.

[51] J. Pascoe, "Adding generic contextual capabilities to wearable computers," in Proc. the Second International Symposium on Wearable Computing, Pittsburgh, PA, October 19-20, 1998.

[52] A. Dey and G. Abowd, "Towards a better understanding of context and context-awareness," in Proc. Workshop on the What, Who, Where, When and How of Context-Awareness at CHI 2000, April 2000.

[53] E. Craig, "Routledge encyclopedia of philosophy: questions to sociobiology," Taylor and Francis, vol. 8, 1998.

[54] A. Nika et al., "A survey of context-aware cross-digital library personalization," Adaptive Multimedia Retrieval Context, Exploration, and Fusion, Springer, pp. 16-30, 2011.

[55] W.-S. Yang and Y.-R. Lin, "A task-focused literature recommender system for digital libraries," Online Information Review, vol. 37, no. 4, pp. 581-601, 2013

[56] Z. Dehghani et al., "A multi-layer contextual model for recommender systems in digital libraries," Aslib Proceedings, vol. 63, no. 6, pp. 555-569, 2011.

[57] W. Woerndl and J. Schlichter, "Introducing context into recommender systems," in Proc. the AAAI Workshop on Recommender Systems in e-Commerce, Vancouver, Canada, 2007.

[58] B. Kaplan and J. A. Maxwell, "Qualitative research methods for evaluating computer information systems," Evaluating the Organizational Impact of Healthcare Information Systems, pp. 30-55. Springer New York, 2005.

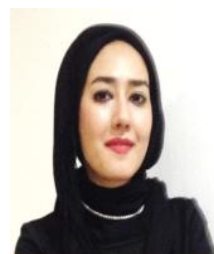

Zohreh Dehghani was born in Isfahan, Iran. She is a $\mathrm{PhD}$ student and received her bachelor and master degrees in information management from the University of Isfahan. She is a senior lecturer in Medical Informatics (MI) Department at the University of Isfahan, Iran since August 2011. Before teaching, Zohre worked as an information service manager, systems analyst and designer for more than five years (2006-2011) in international systems engineering and automation company (IRISA). She was involved in projects that merge human behavior and technology to enable better interactions in various systems such as marketing and interactive systems. Currently, Zohreh is a research assistan in the Human Computer Interaction Lab at the University of Malaya, Malaysia. She is working on Semantic web technologies including linked data and ontologies for recommender systems in digital libraries. Her research interests are human computer interaction, context-aware computing, social networking and information seeking.

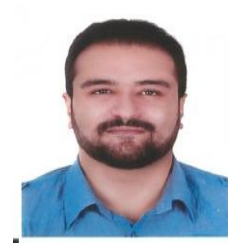

Seyed Reza Shahamiri was born in 1978 in Shiraz. He received the B.S. and M.S. degree in computer engineering - software from Islamic Azad University (IAU), Iran in 2004 and 2007 respectively, and the $\mathrm{Ph} . \mathrm{D}$. in computer science from Universiti Teknologi Malaysia (UTM) in 2011. After graduation, he pursued a one year post-doctoral fellowship at University of Malaya (UM) investigating and developing automatic speech recognition approaches for speech disorders. Dr. Reza was a senior lecturer at Department of Software Engineering, University of Malaya for two years. Currently, he is a senior lecturer at Faculty of Business and Information Technology, Manukau Institute of Technology, Auckland, New Zealand. His research interests include artificial neural networks and pattern recognition, software testing and reliability, automated speech recognition, and software engineering. His research findings have been published in internationally recognized peer-reviewed journals and conference proceedings

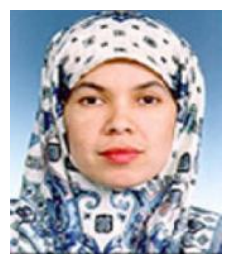

Siti Salwah Salim received her B.Sc. (Hons) from Wichita State University, in the United States and $\mathrm{PhD}$ from the University of Manchester Institute of Science and Technology (UMIST), United Kingdom in 1998. She is currently a professor at Department of Software Engineering, Faculty of Computer Science and Information Technology, University of Malaya, Malaysia. She supervises PhD and master's students in the areas of requirements engineering, component based software development, human computer interaction, automatic speech recognition, and e-learning. She leads the Multimodal Interaction Research Lab for the high impact research grant on multimodal engagement for children with communication disabilities. She has served as a committee member on many editorial boards and research grant screening committees. 